\title{
Simultaneous measurement of refractive index and temperature using a double antiresonant hollow core fiber
}

Ferreira, Marta, Bierlich, Jörg, Kobelke, Jens, Pinto, João, Wondraczek, Katrin

Marta S. Ferreira, Jörg Bierlich, Jens Kobelke, João L. Pinto, Katrin Wondraczek, "Simultaneous measurement of refractive index and temperature using a double antiresonant hollow core fiber," Proc. SPIE 11207, Fourth International Conference on Applications of Optics and Photonics, 112071W (3 October 2019); doi: 10.1117/12.2527473

Event: IV International Conference on Applications of Optics and Photonics (AOP 2019), 2019, Lisbon, Portugal 


\title{
Simultaneous measurement of refractive index and temperature using a double antiresonant hollow core fiber
}

\author{
Marta S. Ferreira ${ }^{* a, b}$, Jörg Bierlich ${ }^{\mathrm{b}}$, Jens Kobelke ${ }^{\mathrm{b}}$, João L. Pinto ${ }^{\mathrm{a}}$, and Katrin Wondraczek ${ }^{\mathrm{b}}$ \\ ${ }^{\mathrm{a}} \mathrm{i}$ N \& Physics Department, University of Aveiro, Campus de Santiago, 3810-193 Aveiro, Portugal. \\ ${ }^{b}$ Leibniz Institute of Photonic Technology, Albert-Einstein-Straße 9, 07745 Jena, Germany.
}

\begin{abstract}
In this work, an inline sensor based on a double antiresonant hollow core fiber is proposed for the simultaneous measurement of refractive index and temperature. The fiber, consisting of four silica capillaries with wall thickness of $\sim 1.5 \mu \mathrm{m}$ and a cladding with a thickness of $\sim 36.5 \mu \mathrm{m}$, is spliced between two sections of single mode fiber. The spectral behavior, measured in transmission, results from the combination of different frequencies which enable the discrimination between the two parameters. The sensing head is subjected to refractive index measurements using aqueous solutions with different concentrations of ethanol. For a sensor with a length of $\sim 10 \mathrm{~mm}$, and considering the lower frequency signal, the sensitivity to refractive index is $389.6 \mathrm{~nm} / \mathrm{RIU}$, whereas for the higher frequency, the sensitivity attained is $2.8 \mathrm{~nm} / \mathrm{RIU}$. On the other hand, the sensing head presented a sensitivity to temperature of $25.5 \mathrm{pm} / \mathrm{K}$ and $-27.6 \mathrm{pm} / \mathrm{K}$ for the higher and lower frequencies, respectively.
\end{abstract}

Keywords: Double antiresonant hollow core fiber, refractive index, temperature, simultaneous measurement.

\section{INTRODUCTION}

The measurement of refractive index of liquids is of great importance for many applications. Several configurations based on optical fiber sensors have been reported in the literature. For instance, the use of fiber tapers ${ }^{1}$, multimode interferometers ${ }^{2}$, modal interferometers based on photonic crystal fibers ${ }^{3}$, Mach-Zehnder interferometers ${ }^{4,5}$, and long period gratings ${ }^{6}$, have been proposed. On the other hand, the liquid infiltration through the holes of microstructured optical fibers ${ }^{7}$, or the creation of access channels through femtosecond laser micromachining ${ }^{8}$ or focused ion beam milling ${ }^{9}$ have also been developed.

In this work, an inline sensor based on an antiresonant microstructured hollow core fiber is proposed. The sensor is used for simultaneous measurement of liquid refractive index and temperature. It should be highlighted that the liquid surrounds the sensor and is not infiltrated, facilitating the measurement and cleaning procedures and reducing the contamination issues. Furthermore, since the sensor outer diameter is similar to standard single mode fiber and the length is of only $\sim 10 \mathrm{~mm}$, the sensor is easy to handle, and low sample volumes are required for the experiments.

\section{SENSOR DESIGN AND SPECTRAL CHARACTERISTICS}

The double antiresonant hollow core fiber (HCF), whose microscopic picture is shown in Fig. 1, was fabricated at the Leibniz Institute of Photonic Technology, Jena (Germany). The fabrication details are described elsewhere ${ }^{10,11}$.

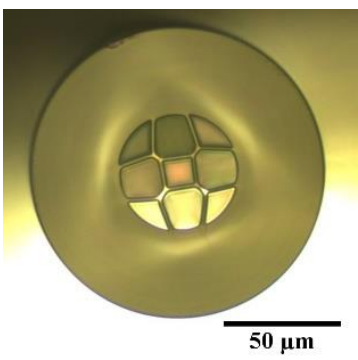

Figure 1 - Microscope image of the antiresonant hollow core fiber cross-section.

*marta.ferreira@ua.pt; phone +351234 370 200; fax +351 234370 985; www.ua.pt

Fourth International Conference on Applications of Optics and Photonics, edited by

Manuel F. M. Costa, Proc. of SPIE Vol. 11207, 112071W · (C) 2019 SPIE

CCC code: $0277-786 X / 19 / \$ 21 \cdot$ doi: $10.1117 / 12.2527473$

Proc. of SPIE Vol. 11207 112071W-1 
The fiber consists of four silica capillaries displayed in diametrically opposed positions, with a wall thickness of $\sim 1.5 \mu \mathrm{m}$. A silica cladding with a thickness of $\sim 36.5 \mu \mathrm{m}$ surrounds the rods. The outer diameter of the HCF is $\sim 125 \mu \mathrm{m}$. The fiber was coated with a standard acrylate during the drawing procedure.

The sensors were fabricated by fusion splicing a section of HCF between two sections of single mode fiber (SMF). To ensure that the fiber structure was not damaged during the splicing procedure, the manual mode of the fusion splicer (Fitel S177) was used, and the fibers were aligned with a lateral offset. In this way, the arc discharge was mainly applied to the SMF section. Furthermore, low arc power and discharge time were set, of 13 arb. units and $300 \mathrm{~ms}$, respectively. One side of the sensor was connected to a supercontinuum optical source (Fianium, WL-SC-400-2), while the other side was connected to an optical spectrum analyzer (OSA Anritsu MS9740A, with a resolution of $0.02 \mathrm{~nm}$ ), in a typical transmission scheme.

Figure 2 (left) shows the transmission spectra for a sensing head with a length of $10.4 \mathrm{~mm}$, when surrounded by air and when submerged in water. In the first case, several lossy dips can be observed, which satisfy the condition ${ }^{10}$

$$
\lambda_{m}=\frac{2 t}{m} \sqrt{n^{2}-1}
$$

where $\lambda_{m}$ is the dip wavelength, $t$ is the thickness of the cladding, $m$ an integer and $n$ the refractive index of silica. When the sensor is submersed in water, these resonant modes are filtered, and the spectral behavior changes considerably.
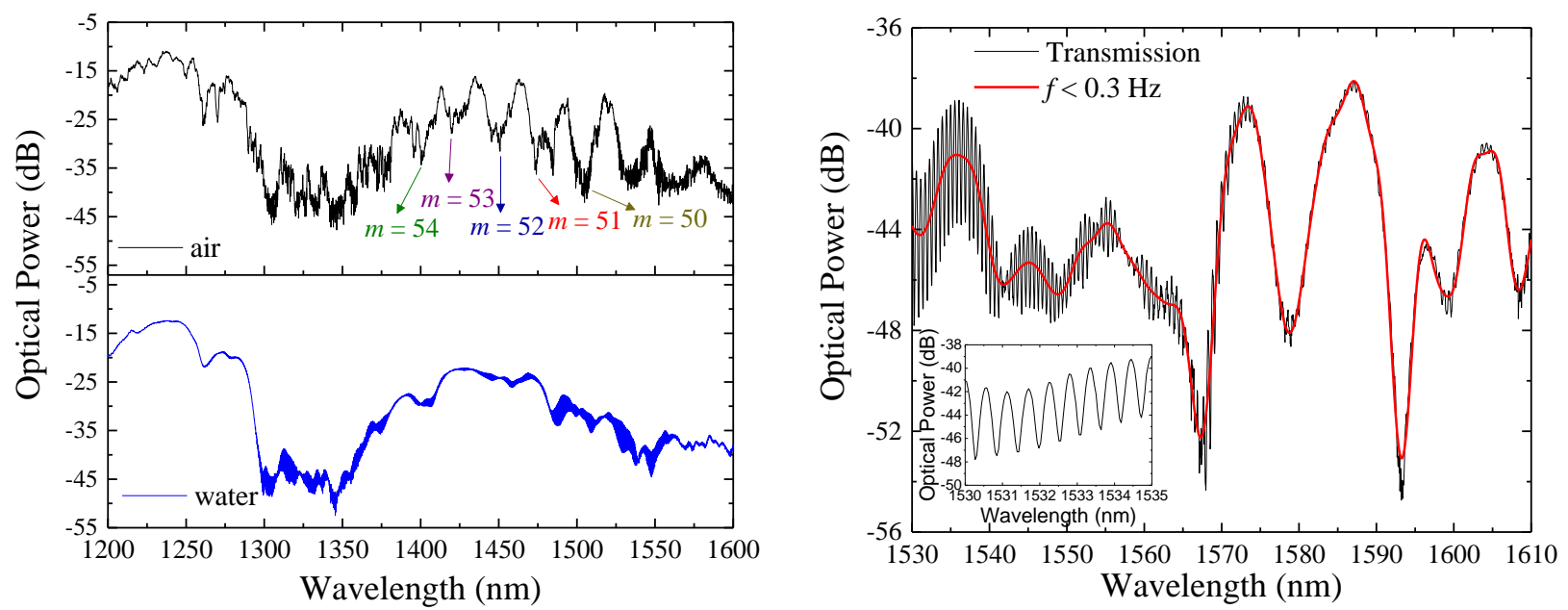

Figure 2 - (left) Transmission spectra of one sensing head with a length of $10.4 \mathrm{~mm}$, when surrounded by air (top) and when submerged in water (bottom). (right) Transmission spectrum for the sensing head with a length of $9.9 \mathrm{~mm}$. Inset: Zoom-in of the spectrum in the $1530-1535 \mathrm{~nm}$ region, to highlight the high frequency interference.

Figure 2 (right) shows the transmission spectrum of a sensor submerged in water, with a length of $9.9 \mathrm{~mm}$, in a range between $1530 \mathrm{~nm}$ and $1610 \mathrm{~nm}$. A high frequency signal is observed (see inset Fig. 2 (right)), which, considering the sensor length and the wavelengths of two adjacent peaks, presents an effective refractive index of $0.44 \mathrm{RIU}$. This means that the signal is originated by the interference between a mode propagating in air and another propagating in silica, constituting a Mach-Zehnder interferometer. On the other hand, this signal is modulated by a low-frequency signal, highlighted by the red line in the curve (obtained through an FFT low bandpass filter). This modulation arises from the interference between modes propagating in the outer cladding region, close to the external medium and it is sensitive to variations of liquid refractive index.

\section{RESULTS}

A series of ethanol-water mixtures were prepared under a controlled laboratory environment and stored for $24 \mathrm{~h}$ to allow their stabilization. After the stabilization period, the refractive index of each solution was measured using an Abbe refractometer (KRÜSS optronic refractometer) operating at $589 \mathrm{~nm}$. The sensor was placed in a support structure, and it was kept straight throughout the experiments, to minimize the optical losses due to fiber bending. A small amount of each solution was placed in direct contact with the sensing structure, and the wavelength shift of both low and high frequencies (MZI) was measured. The results, presented in Fig. 3, show that the responses for each frequency are very 
different. For the high frequency the sensitivity was of $2.8 \mathrm{~nm} / \mathrm{RIU}$, whereas for the low frequency signal a slightly nonlinear behavior was observed. However, if we consider a linear fitting, a sensitivity of $389.6 \mathrm{~nm} / \mathrm{RIU}\left(r^{2}=0.991\right)$ can be estimated, as a first approximation.

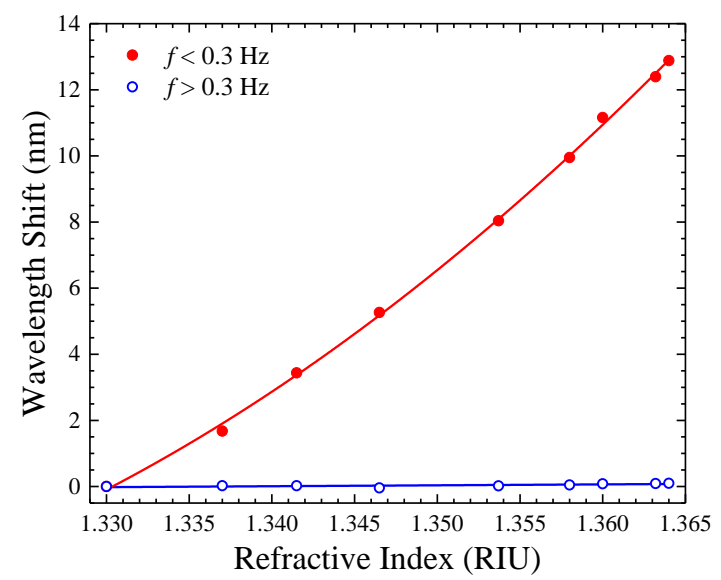

Figure 3 - Sensor response to liquid refractive index variations.

The sensor response to temperature variations was evaluated by placing the sensor straight in a water filled container placed on a hot plate and by varying the temperature from room temperature up to $65^{\circ} \mathrm{C}$. In this case, the high frequency signal originated by the MZI presented a linear sensitivity of $25.5 \mathrm{pm} / \mathrm{K}$, whereas the low frequency signal presented a shift towards smaller wavelengths (blue shift), with a sensitivity of $-27.6 \mathrm{pm} / \mathrm{K}$. This behavior is correlated with the thermo-optic coefficient of water, which, in this case is the dominant effect over thermal expansion.

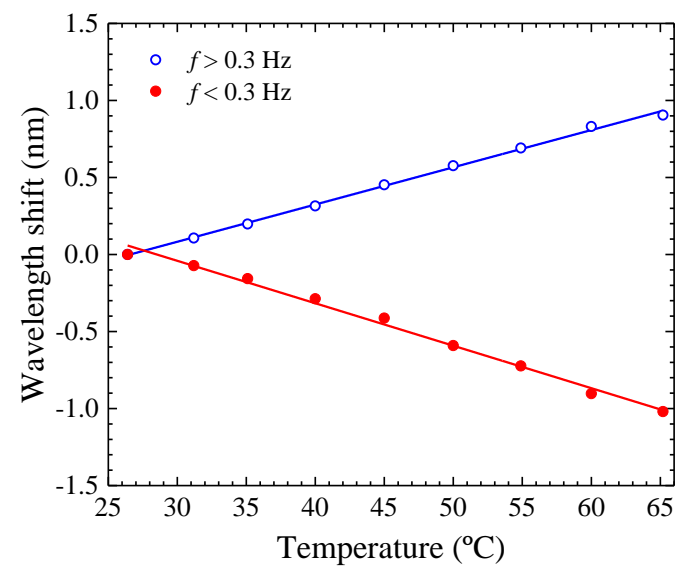

Figure 4 - Sensor response to water temperature variations.

Given the different sensitivity coefficients towards refractive index and temperature, this sensor can be a good candidate to perform simultaneous measurement of the two measurands. In order to determine the refractive index and temperature variations from the low frequency $(L F)$ and high frequency $(H F)$ signals, the following matrix can be employed:

$$
\left[\begin{array}{c}
\Delta n \\
\Delta T
\end{array}\right]=\frac{1}{D}\left[\begin{array}{cc}
k_{T H F} & -k_{T L F} \\
-k_{n H F} & k_{n L F}
\end{array}\right]\left[\begin{array}{c}
\Delta \lambda_{L F} \\
\Delta \lambda_{H F}
\end{array}\right]
$$

Where $D=k_{T H F} k_{n L F}-k_{T L F} k_{n H F}$ is the matrix determinant, and $k_{T H F}, k_{T L F}, k_{n L F}$, and $k_{n H F}$ are the temperature and refractive index sensitivities, respectively. Inserting the previously calculated sensitivity coefficients yields:

$$
\left[\begin{array}{c}
\Delta n \\
\Delta T
\end{array}\right]=\frac{1}{10.01}\left[\begin{array}{cc}
25.5 \times 10^{-3} & 27.6 \times 10^{-3} \\
-2.8 & 389.6
\end{array}\right]\left[\begin{array}{l}
\Delta \lambda_{L F} \\
\Delta \lambda_{H F}
\end{array}\right]
$$

where the $\Delta n, \Delta T$, and $\Delta \lambda$ units are RIU, $\mathrm{K}$, and $\mathrm{nm}$, respectively. 


\section{CONCLUSIONS}

In summary, an inline sensor based on an antiresonant hollow core fiber was proposed for the simultaneous measurement of refractive index and temperature. When submerged in liquid media, the spectral behavior exhibits a high frequency and a low frequency signals, which exhibit different responses to both measurands. For a $9.9 \mathrm{~mm}$ long sensing head, a sensitivity to refractive index of $389.6 \mathrm{~nm} / \mathrm{RIU}$ was attained for the low frequency signal, whereas for the higher frequency, the sensitivity obtained was of is $2.8 \mathrm{~nm} / \mathrm{RIU}$. On the other hand, the sensing head presented a sensitivity to temperature of $25.5 \mathrm{pm} / \mathrm{K}$ and $-27.6 \mathrm{pm} / \mathrm{K}$ for the higher and lower frequencies, respectively. The proposed sensing device is easy to manufacture, and to handle. Besides, since the liquid does not enter the fiber, the cleaning is straightforward, and the contamination issues are minimized. To the best of our knowledge, this is the first time that an anti-resonant microstructured hollow core fiber is proposed for simultaneously measuring the refractive index and temperature of liquids, without the need to infiltrate them in the hollow structure.

\section{ACKNOWLEDGMENTS}

Funding from the federal state of Thuringia (FKZ: 2012 FGR 0013; FKZ: 2016 FGR 0051) and ESF is highly acknowledged. This work was supported by FEDER funds through the COMPETE 2020 Programme and National Funds through FCT-Portuguese Foundation for Science and Technology under the Projects UID/CTM/50025/2013 and AROMA (PTDC/EEI-EEE/31568/2017).

\section{REFERENCES}

[1] Yadav, T. K., Narayanaswamy, R., Abu Bakar, M. H., Mustapha Kamil, Y. and Mahdi, M. A., "Single mode tapered fiber-optic interferometer based refractive index sensor and its application to protein sensing," Opt. Express 22(19), $22802-22807$ (2014).

[2] Novais, S., Ferreira, C. I. A., Ferreira, M. S., Pinto, J. L., "Optical fiber tip sensor for the measurement of glucose aqueous solutions," IEEE Photonics J. 10(5), 6803609 (2018).

[3] Jha, R., Villatoro, J., and Badenes, G., "Ultrastable in reflection photonic crystal fiber modal interferometer for accurate refractive index sensing," Appl. Phys. Lett. 93, 191106 (2008).

[4] Pang, F., Liu, H., Guo, H., Liu, Y., Zeng, X., Chen, N., Chen, Z., and Wang T., "In-fiber Mach-Zehnder interferometer based on double cladding fibers for refractive index sensor," IEEE Sensors J. 11(10), 2395-2400 (2011).

[5] Harris, J., Lu, P., Larocque, H., Chen, L., and Bao, X., "In-fiber Mach-Zehnder interferometric refractive index sensors with guided and leaky modes," Sensor. Actuat. B-Chem 206, 246-251 (2015).

[6] Esposito, F., Ranjan, R., Campopiano, S. and Iadicicco, A., "Experimental study of the refractive index sensitivity in arc-induced long period gratings," IEEE Photonics J. 9(1), 7100110 (2017).

[7] He, Z., Zhu, Y., and Dua, H., "Long-period gratings inscribed in air- and water-filled photonic crystal fiber for refractometric sensing of aqueous solution," Appl. Phys. Lett. 92, 044105 (2008).

[8] Zhou, K., Yan, Z., Zhang, L., Bennion, I., "Refractometer based on fiber Bragg grating Fabry-Pérot cavity embedded with a narrow microchannel," Opt. Express 19(12), 11769 - 11779 (2011).

[9] Zheng, Y., Chen, L. H., Yang, J., Raghunandhan, R., Dong, X., So, P. L., Chan, C. C., "Focused ion beam ablated fiber optic optofluidic sensor for fast refractive index and magnetic field measurement," IEEE J Sel. Top. Quant. 23(2), 5601905 (2017)

[10] Hartung, A., Kobelke, J., Schwuchow, A., Wondraczek, K., Bierlich, J., Popp, J., Frosch, T. and Schmidt, M. A., "Double antiresonant hollow core fiber-guidance in the deep ultraviolet by modified tunneling leaky modes," Opt. Express 22(16), 19131-19140 (2014).

[11] Schuster, K., Unger, S., Aichele, C., Lindner, F., Grimm, S., Litzkendorf, D., Kobelke, J., Bierlich, B., Wondraczek, K. and Bartelt, H., "Material and technology trends in fiber optics," Adv. Opt. Techn. 3(4), 447-468 (2014). 